\title{
Gene regulatory landscape in osteoblast differentiation
}

\author{
Hironori Hojo ${ }^{1}$ and Shinsuke Ohba ${ }^{2 *}$
}

${ }^{1}$ Department of Clinical Biotechnology, Center for Disease Biology and Integrative Medicine, The University of Tokyo Graduate School of Medicine, 7-3-1 Hongo, Bunkyo-ku, Tokyo 113-8655, Japan

${ }^{2}$ Department of Cell Biology, Institute of Biomedical Sciences, Nagasaki University, 1-7-1

Sakamoto, Nagasaki 852-8588, Japan

*Corresponding author: Shinsuke Ohba, Department of Cell Biology, Institute of Biomedical Sciences, Nagasaki University, 1-7-1 Sakamoto, Nagasaki 852-8588, Japan

Telephone: +81-95-819-7630; Fax:+81-95-819-7633; email: s-ohba@nagasaki-u.ac.jp

Keywords: osteoblasts; transcriptional regulators; gene regulatory landscape; gene regulatory networks; epigenome; epigenetics 


\begin{abstract}
The development of osteoblasts, a bone-forming cell population, occurs in conjunction with development of the skeleton, which creates our physical framework and shapes the body. In the past two decades, genetic studies have uncovered the molecular framework of this process - namely, transcriptional regulators and signaling pathways coordinate the cell fate determination and differentiation of osteoblasts in a spatial and temporal manner. Recently emerging genome-wide studies provide additional layers of understanding of the gene regulatory landscape during osteoblast differentiation, allowing us to gain novel insight into the modes of action of the key regulators, functional interaction among the regulator-bound enhancers, epigenetic regulations, and the complex nature of regulatory inputs. In this review, we summarize current understanding of the transcriptional regulation in osteoblasts, in terms of the gene regulatory landscape.
\end{abstract}




\section{Introduction}

The mammalian skeleton is derived from three distinct origins and formed through two distinct modes [1]. The neural crest gives rise to the facial bones and the cranium. The paraxial mesoderm gives rise to the parietal bone, base of the skull, and axial skeleton. The lateral plate mesoderm gives rise to the appendicular skeleton. The two distinct modes of skeletal formation are intramembranous ossification and endochondral ossification. Skull and facial bones develop through intramembranous ossification, where condensed mesenchymal cells directly differentiate into bone-forming osteoblasts. The remainder of the skeleton develops through endochondral ossification. In this process, a cartilage mold is first formed following condensation of mesenchymal cells and then replaced by mineralized bone tissues.

Specification and differentiation of two cell linages, chondrocytes and osteoblasts, are coordinated during endochondral ossification. Cells in the center of the condensed mesenchyme differentiate into proliferating chondrocytes and subsequently into pre-hypertrophic chondrocytes and hypertrophic chondrocytes. Hypertrophic chondrocytes finally undergo calcification, forming mineralized cartilages. Osteoblast progenitors exist in the perichondrium, a thin layer of cells that are originated in the condensed mesenchyme and located at the periphery of cartilage. Once the progenitors receive osteogenic inputs - mainly from the factors secreted by hypertrophic chondrocytes - they are specified into osteoblast precursors $[2,3]$. They mature into osteoblasts that deposit the bone matrix forming the bone collar. Finally, the osteoblasts are embedded in the matrix and work as osteocytes. The osteoblast precursors also invade into the mineralized cartilage with vascular tissues to replace the mineralized cartilage with bone matrix [4].

In the osteoblast linage, alkaline phosphatase (Alpl) and integrin binding sialoprotein (Ibsp) are expressed in both osteoblast precursors and osteoblasts. High expression of type I collagen alpha I (Col1a1) and secreted phosphoprotein 1 (Spp1, also known as osteopontin) is restricted to osteoblasts, although Col1a1 is also ubiquitously expressed at a low level in fibroblastic cells. Bone 
gamma-carboxyglutamate protein (Bglap, also known as osteocalcin) is a bona-fide marker for mature osteoblasts. Osteocytes are characterized by the expression of dentin matrix protein 1 (Dmp1), which is also expressed in odontoblasts of teeth, and sclerostin (Sost). The importance of sclerostin in bone metabolism has been highlighted by several basic studies and the use of antisclerostin antibodies for the treatment of osteoporosis [5]. Thus, the stage-specific expression of marker genes enables us to see the process of osteoblast differentiation in its chronological order, beginning with the epiphysis and continuing toward the diaphysis in the perichondrial region of developing long bones.

Two transcription factors (TFs), Runx2 and Sp7/Osterix, are required for osteoblast specification and act as master regulators of osteoblast development. Runx2 is expressed in perichondrial cells adjacent to columnar proliferating chondrocytes as well as osteoblasts in the bone collar and primary spongiosa in endochondral bones, whereas $S p 7$ expression is more restricted to cells associated with the bone collar and primary spongiosa [6, 7]. In Runx $2^{-/-}$mice and $S p 7^{-/-}$mice, both intramembranous and endochondral bones are absent; no functional osteoblast develops in both mutants [7, 8]. Heterozygous loss-of-function mutations of RUNX2 in humans cause cleidocranial dysplasia, an autosomal dominant disease characterized by hypoplasia/aplasia of the clavicle, patent fontanelles, supernumerary teeth, short stature, and other changes in skeletal patterning and growth [9-11]. SP7 mutations have been associated with a recessive osteogenesis imperfecta $[12,13]$.

These two master regulators sequentially act on osteoblast precursors in the process of osteoblast differentiation. Skeletal progenitors are initially committed to Runx2-positive osteoblast precursors, which then transition into Runx2- and Sp7-double-positive precursors [14]. The Runx2 and Sp7-double-positive precursors and their descendent give rise to osteoblasts and osteocytes, contributing to the formation of cortical bones, trabecular bones, and bone marrow with invading blood vessels [4]. The relationship between Runx2 and Sp7 is also genetically supported. Runx2 is expressed in $S p 7^{-/-}$mice, whereas no $S p 7$ expression is observed in $R u n x 2^{-/-}$mice [7]. 
The process of cell fate specification and differentiation of osteoblasts have been extensively studied through mouse genetics and biochemical approaches for the last two decades; it turns out that the process is well organized by sequential and/or combinatorial actions of TFs, including Runx2 and Sp7/Osterix, and signaling pathways (extensively reviewed in [15]). However, those studies focused on local gene regulatory networks. Extensive next generation sequencer (NGS)-based studies have uncovered modes of actions of the key TFs, functional interaction among TF-bound enhancers, epigenetic regulations, and the complex nature of regulatory inputs over the osteoblast genome. The NGS-based analyses include chromatin immunoprecipitation sequencing (ChIP-seq; profiling of TF binding sites and histone modifications), RNA sequencing (RNA-seq; profiling of gene expression), and an assay for transposase-accessible chromatin sequencing (ATACseq; profiling of open chromatin regions), and so on.

In this review, with a particular focus on osteoblasts, we discuss modes of action of master regulators and epigenetic regulations, in terms of the gene regulatory landscape recently revealed by unbiased genome-wide studies.

\section{Overview of gene regulation}

The genome coordinates developmental processes through multiple layers of gene regulation. The model is evolved from the concept of the "epigenetic landscape," which was proposed by C.H. Waddington in 1957 [16]. In this concept, a cell chooses its fate by following the contours of the developmental landscape, just as a ball rolls down through valleys. In 1969, R.J. Britten and E.H. Davidson then introduced the theory of the gene regulatory network, in which the DNA sequencespecific regulation of gene expression underlies the genomic control of developmental processes [17]. Thus, a deeper understanding of the gene regulatory network will be key to elucidating how the genome encodes and executes the program to specify cell fates, induce differentiation of the specified cells, and lead them to exert biological functions in development and maintenance of 
tissues.

Gene expression is regulated by the interaction between the basal promoter and enhancers. The basal promoter recruits basal transcription machineries and RNA polymerase to initiate transcription. Although the basal promoter is required for the transcriptional initiation, it is not sufficient to achieve the transcription of a proper set of genes in proper cells with the proper timing. Additional DNA regulatory elements known as enhancers provide the spatiotemporal regulation of gene expression. A 72-bp repeated element in SV40 DNA was first identified as an enhancer in 1981; the element acted as a cis-regulatory element, which activated transcription regardless of its orientation at several positions [18]. Thus, enhancers are currently defined as DNA sequences that have potential to mediate the spatial and temporal pattern of gene expressions regardless of the orientation of the sequence, i.e., forward or reverse, and the location of the sequence. They can function at mega-base distances from the transcription start sites (TSSs), as exampled by a longrange interaction to regulate Shh expression [19]. Trans-acting factors such as TFs and their cofactors bind to enhancers in a sequence-specific manner. The binding regulates the transcription of target genes in a spatiotemporally specific manner through the promoter-enhancer interaction.

\section{The mode of Runx 2 action in osteoblasts}

Runx 2 belongs to the runt-related transcription factor family. Runx 2 recognizes the Runx consensus motif on the genome through its DNA-binding domain, the runt domain. The initial link between Runx2 and osteoblasts was shown by a series of reports which described that it bound to an osteoblast-specific cis-regulatory element in the promoter region of the Bglap gene [6, 20]. Runx2associated cis-regulatory elements had been identified in analyses of non-coding regions flanking osteoblast genes such as Alpl, Ibsp and Spp1 [21]. Three independent groups performed Runx2 ChIPseq studies, providing the four key findings on the Runx2 regulatory landscape in the osteoblast program [22-24]. 
First, the actions of Runx 2 on the genome are dynamically changed during osteoblast differentiation. Peak distribution analysis showed that Runx2 mainly binds to distal genomic regions at least $1 \mathrm{~kb}$ distant from TSSs, indicating that distal interaction is a general feature of the Runx2 action. Gene ontology analyses suggested the complexities of Runx2 functions. Wu et al. performed clustering analysis of the Runx2 ChIP-seq peak distribution and its intensities across various time points in an in vitro osteoblast induction culture [24]. Only a subset of Runx2 peaks, which were highly associated with genes related to osteoblast differentiation, appeared upon osteoblast induction; known Runx2 targets such as Runx2 itself, Ibsp, and Sp7 are included in this cluster [24]. In contrast, another cluster of Runx2 peaks whose intensities are lost upon the induction is related to biological functions of other lineages, including fat cell differentiation, leukocyte migration, and erythrocyte differentiation [24]. These findings suggest that Runx2 has broader and less-specific actions than Sp7 (also see the $\mathrm{Sp} 7$ section in this review). As Wu et al. discussed, Runx2 may not only induce osteoblast differentiation of progenitors, but also suppress differentiation into non-osteogenic cell lineages by masking genomic regions related to different cell lineages [24]. Although we cannot exclude the possibility that in vitro culture may not be biologically relevant to the osteoblast progenitor stage, we speculate that as osteoblast differentiation proceeds, the Runx2-madiated masking may be no longer required and somehow depleted, while Sp7 acting downstream of Runx2 is restricted to osteoblast enhancers.

Second, new Runx2-bound cis-regulatory regions were identified [22-24]. The distal cisregulatory elements flanking Mmp13 and Spp1 were notable, given that Runx2 had been reported to bind to the proximal elements of these genes $[25,26]$. The newly found distal cis-regulatory elements had more intense Runx2 binding signatures than the proximal elements [23, 27]. In addition, the distal Mmp13 enhancer showed strong responsiveness to Runx2, and the deletion of the distal enhancer using CRISPR/Cas9 dramatically suppressed Mmp13 expression in vitro [27].

Third, a Runx consensus motif was most enriched in Runx2 ChIP-seq peaks, indicating that 
Runx2 predominantly functions through this motif $[22,23]$ (Fig. 1). This finding is consistent with a large number of previous studies supporting the idea that Runx2 binds to the motif. Meyer et al. further identified $\mathrm{C} / \mathrm{EBP} \beta$ as a co-regulator of Runx2 on the osteoblast genome, based on the high enrichment of the $\mathrm{C} / \mathrm{EBP} \beta$ motif in the Runx2 ChIP-seq data and the subsequent C/EBP $\beta$ ChIP-seq [23]. Since Runx 2 and C/EBP $\beta$ form a transcriptional complex and function in transactivation [28, 29], they are likely to share osteoblast enhancer modules (Fig. 1). In addition to the aforementioned study, Meyer et al. also performed ChIP-seq for six TFs (Runx2, C/EBP $\beta, \mathrm{C} / \mathrm{EBP} \alpha$, PPAR $\gamma$, vitamin D receptor (VDR), and retinoid X receptor (RXR)) in the osteogenic differentiation of mouse MSCs. Osteoblast-related genes were accompanied by the binding of Runx2, C/EBP $\beta, \mathrm{VDR}$, and RXR, suggesting the presence of TF "hot spots" around key genes for osteoblast identity [30] (Fig. 1). Hotspots are genomic regions where key transcriptional regulators co-bind to form core regulatory networks, defining cell identity [31] (Fig. 1). It remains to be elucidated where the bona-fide hotspot is on the osteoblast genome.

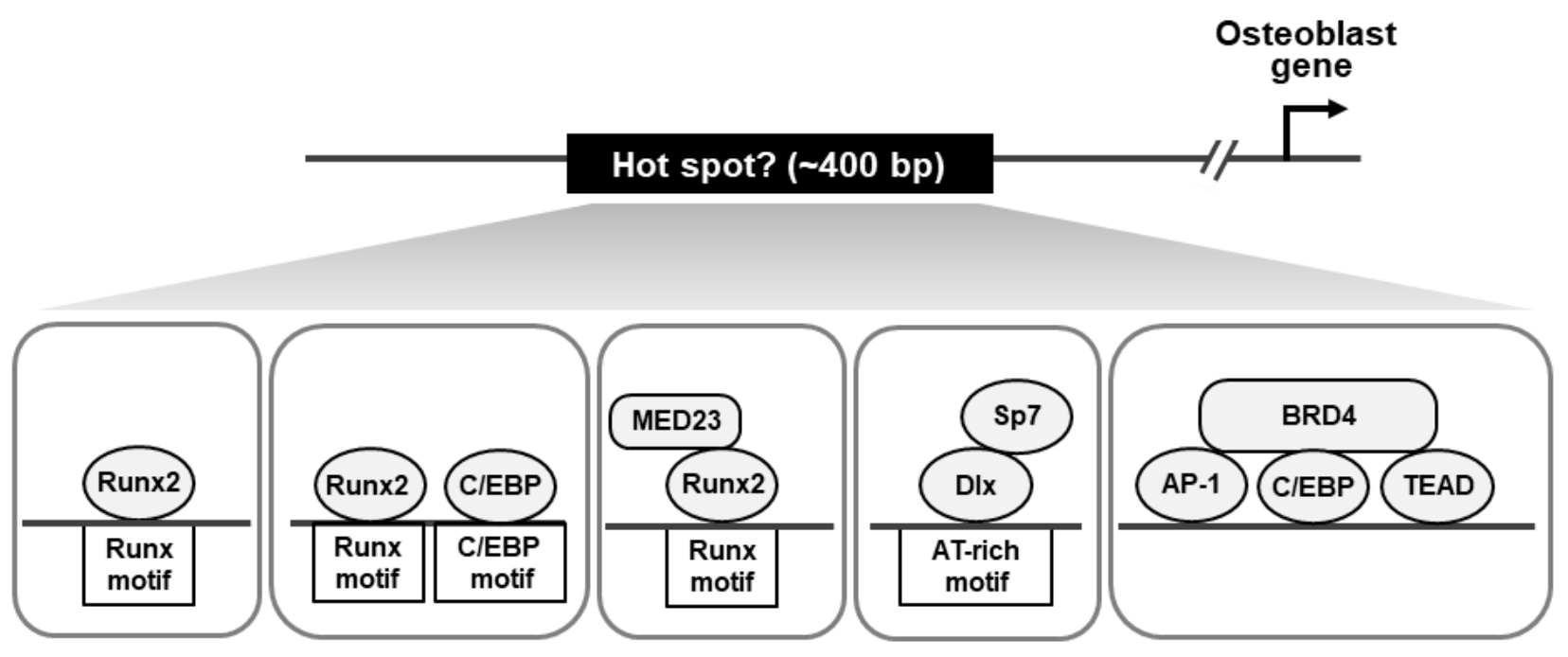

Fig. 1. Global actions of transcription factors (TFs), cofactors, and epigenetic regulators in osteoblasts.

Multiple TFs, cofactors, and epigenetic regulators are thought to co-localize at small genomic regions termed transcription hot spots $(\sim 400 \mathrm{bp})$ to specify osteoblasts. Key interactions obtained from ChIPseq studies are illustrated. 
In addition to $\mathrm{C} / \mathrm{EBP} \beta$, a number of transcriptional regulators have been identified as cooperative factors of Runx 2 by in vitro and in vivo studies, including Cbfb [32, 33], Twist [34], Stat1 [35], Schnurri 3 [36], SATB2 [37], TAZ [38], MED23 [39] (also see the epigenetic landscape section in this review), Nell-1 [40], and Zfp521 [41]. Further interaction studies by genome-wide approaches will help to clarify their biological relevance from the viewpoint of the gene regulatory landscape.

Lastly, epigenetic regulation may make only a small contribution to the Runx2-DNA interaction. Integrated bioinformatic analysis on epigenome, transcriptome, and Runx2-DNA binding profiles identified dynamic signatures of histone modifications and transcriptional profiles during osteoblast differentiation [23, 24]. Given that Runx2 functions in a differentiation stage-dependent manner, one can imagine that the epigenetic change determines the stage-specific Runx 2 function in osteoblast differentiation. However, Runx2 binding profiles were not correlated well with the dynamic signatures of histone modifications and transcription. Although in vitro settings may be less biologically relevant, the results suggest that Runx2-DNA interactions are stable during osteoblast differentiation; other factors may modify Runx2 functions without altering Runx2-DNA interactions. Further analyses will be needed before drawing conclusions regarding this argument. The epigenetic landscape in osteoblasts will be discussed later.

Mesenchymal stem cells (MSCs) are shown to be a common source for osteoblasts and adipocytes in the bone marrow. As Runx 2 promotes osteoblast differentiation, one can imagine that it in turn blocks adipocyte differentiation in terms of cell fate determination of MSCs. Meyer et al. provided some clues to understanding the role of Runx2 in a genome-wide manner. Runx2 ChIP-seq in mouse MSCs under either osteogenic and adipogenic conditions revealed that Runx2-binding regions in adipocyte-directed cells were less than those in osteoblast-directed cells [30]. In addition, they found TF "hot spots," where approximately $30 \%$ of Runx 2 peaks and $35 \%$ of PPAR $\gamma$ peaks 
were overlapped [30], suggesting that Runx2 is somehow involved in adipogenesis together with PPAR $\gamma$. Given that postnatal Runx2 deficiency led to bone marrow adiposity in mice, Runx2 may have negative impacts on PPAR $\gamma$-mediated adipocyte differentiation [42]. However, the work by Rauch et al. did not highlight Runx2 as a suppressor of adipogenesis [43]. They predicted a set of TFs as suppressors of adipogenesis using a computational method called IMAGE, based on transcriptome and enhancer activity data in osteogenic or adipogenic cultures of human MSCs, and confirmed the suppressor function of predicted TFs by knockdown experiments; Runx2 was not included in the predicted set of TFs [43]. Thus, mechanisms underlying the involvement of Runx2 in adipogenesis are still debated.

\section{The mode of Sp7/Osterix action in osteoblasts}

$\mathrm{Sp7/Osterix} \mathrm{is} \mathrm{classified} \mathrm{into} \mathrm{the} \mathrm{Sp} \mathrm{family} \mathrm{of} \mathrm{transcription} \mathrm{factors} \mathrm{and} \mathrm{has} \mathrm{been} \mathrm{thought} \mathrm{to} \mathrm{function}$ through its binding to the Sp consensus motif, GC-box, on the genome [7]. Our Sp7 ChIP-seq study provided further novel insight into the $\mathrm{Sp} 7$ regulatory landscape, the mode of Sp7 action, and an evolutionary relationship between $\mathrm{Sp} 7$ and bone-forming vertebrates [44].

The Sp7-DNA association profile was obtained by Sp7 ChIP-seq in in vivo mouse primary osteoblasts. Distribution analysis of Sp7 ChIP-seq peaks revealed that longer-range interactions $(>5$ $\mathrm{kb})$ were the predominant feature of the $\mathrm{Sp} 7$ action [44]. The sequences of $\mathrm{Sp} 7$ peaks were well conserved among different vertebrate species, and the peak regions were strongly associated with genes that were related to skeletal development and expressed in bone tissues [44]. The biological relevance of $\mathrm{Sp} 7$ peaks was confirmed by correlation analysis with transcriptome data obtained from calvarial osteoblasts of neonatal Sp7-GFP reporter mice [14].

$\mathrm{Sp} 7$ is known to have a highly conserved zinc finger domain. Sp family members bind to the GC-box consensus motif, which is mainly located in promoter regions on the genome, via their zinc finger domains [45]. ChIP-seq analysis for Sp1, Sp2, and Sp5 [46-48] and high throughput screening 
of protein-DNA bindings for Sp1, Sp3, and Sp4 $[49,50]$ have confirmed the mode of action. In contrast, de novo motif analysis demonstrated the enrichment of an AT-rich motif, not the GC-box, in the Sp7 ChIP-seq peak regions [44]. The AT-rich motif resembled a homeodomain-responsive element.

This unexpected finding was supported by comparative analysis of Sp1 and Sp7 ChIP-seq in the pre-osteoblastic cell line MC3T3-E1; the AT-rich motif, but not the GC-box, was again enriched in Sp7 peaks, whereas Sp1 favored the GC-box [44]. Peak distribution analysis of the two data sets revealed that the difference in genomic occupancy might underlie the difference in functions of these two Sp members; Sp1 was mainly associated with proximal regions from TSSs of housekeeping genes, whereas Sp7 was mainly associated with distal genomic regions of osteoblast-related genes [44]. These results indicate that $\mathrm{Sp} 7$ has a mode of action distinct from those of other $\mathrm{Sp}$ family members, in terms of both genomic targets and the binding manner.

Consistent with the data of the de novo motif analysis, Sp7 binding affinity to the GC-box was much less than that of Sp1 and, in most cases, below the detection limit in biochemical analyses [44]. Interestingly, three amino acid substitutions in the Sp7 zinc fingers to their Sp1 counterparts reverted $\mathrm{Sp} 7$ back to a GC-box-binding factor, suggesting that the amino acid differences within the zinc finger domain were responsible for the loss of the GC-box preference in Sp7.

In biochemical analyses, direct binding of $\mathrm{Sp} 7$ to the AT-rich motifs was not detected [44]. How does Sp7 interact with the AT-rich motifs on the osteoblast genome? Several lines of evidence suggested that $\mathrm{Sp} 7$ was indirectly associated with the AT-rich motif on the genome through physical interaction with homeodomain-containing Dlx transcription factors. First, Dlx factors are highly expressed in osteoblasts and have been reported to contribute to osteoblast differentiation [51-53]. Second, comparison of Dlx5 ChIP-seq peaks with Sp7 ChIP-seq peaks in MC3T3-E1 revealed that almost $80 \%$ of Sp7-bound regions overlapped with Dlx5-bound regions [44]. Third, Sp7 physically interacted with Dlx5; the Sp7 zinc finger domain and the Dlx5 N-terminal domain were crucial for the interaction [44]. Fourth, the Sp7-Dlx5 complex bound to the AT-rich motif, whereas Sp7 alone did not, 
as mentioned earlier [44]. Lastly, knock-down of Dlx factors in osteoblasts attenuated $\mathrm{Sp} 7$ engagements with the osteoblast enhancers on the genome, resulting in suppression of the Sp7 target gene expression [44]. These data suggest that $\mathrm{Sp} 7$ prefers to act as a Dlx co-factor rather than as a TF, in order to execute its osteoblast program (Fig. 1). However, it remains to be investigated whether Sp7 acts as a co-factor only for Dlx factors. Other homeodomain-containing TFs, such as Msx1/2, Satb2 and Alx4 are all highly expressed in osteoblasts [44]. Based on motif databases, those factors may bind to the AT-rich motif in a similar manner [50] and may interact with Sp7. In addition, motif analysis on Sp7 ChIP-seq data also showed the enrichment of consensus motifs for Runx2 [54], Nfatc1 [55], and other key bone regulatory factors, suggesting the integration of several regulatory inputs through the identified cis-regulatory modules.

Given that key amino acid variants in Sp7 were responsible for the lack of its GC-box preference, the appearance of Sp7 was examined in the evolution of Sp-family members and bone formation [44]. The closest non-boney vertebrates (e.g., lampreys), the cephalochordates (e.g., amphioxus), and ascidians (e.g., tunicate) all lack Sp7-type zinc finger variants in their genome, whereas $\mathrm{Sp} 7$ is present in all boney vertebrates, including cartilaginous fish, which arise from a boney ancestor and show evidence of non-endochondral bone [44]. These data support the view that the appearance of Sp7-like variants in the Sp family is closely coupled to the emergence of boneforming osteoblasts in the evolution of boney vertebrates. Sp7 may be a key evolutionary switch in the cartilage-to-bone transition. As Dlx paralogs are present in non-vertebrate chordates, Sp7 may emerge as a stabilizer or enhancer of the pre-existing Dlx functions in distinct targets to execute the osteoblast program.

The Sp7 ChIP-seq study also identified novel genomic targets of Sp7 in osteoblasts. The ChIP-seq data demonstrated Sp7 engagement with known osteoblast enhancers flanking Runx2 [56] and Col1a1 [57] as well as Sp7 engagement with a novel enhancer in Notch2 intron 1 [44]. Sitedirected mutations in AT-rich motifs within these elements suppressed their enhancer activities [44], 
suggesting that $\mathrm{Sp} 7$ acts on the elements through the AT-rich motif. The target gene analysis also suggested cross-talk between Sp7 and osteogenic signaling pathways such as Notch, Hedgehog, fibroblast growth factor (FGF), and canonical Wnt signaling. Sp7 ChIP-seq peaks were found around Notch2, Gli2, Fgfr2, and the Kremen1 [44]. These data also suggest that Sp7 is involved in fine tuning of these signaling pathways at the transcriptional level in the osteoblast program. In this context, Sp7 may also interact with Runx2 to execute the program. Several studies have shown their physical interaction and synergistic functions in the transcription of osteoblast-related genes [58, 59]. In addition, de novo motif analysis in our Sp7 ChIP-seq demonstrated the enrichment of a Runx motif as well as the AT-rich motif [44], suggesting the interaction between Sp7 and Runx2 on the osteoblast genome. Integrative analysis of Runx2 ChIP-seq, Sp7 ChIP-seq, and transcriptional profiling will further unravel the mode of the interaction and its biological significance in a genomewide manner.

As mentioned in the section of Runx2, one may also predict that $\mathrm{Sp} 7$ has negative impacts on adipogenesis. However, none of in vivo or genome-wide data has clearly supported the prediction so far, although gain of function of $\mathrm{Sp} 7$ was shown to repress adipogenesis in the mouse adipocyte cell line 3T3-L1 [60].

\section{Epigenetic landscape in osteoblasts}

TFs recognize their consensus motifs on DNA. However, it should be noted that not all consensus motifs of a TF are occupied on the genome even if that TF is highly expressed; TFs bind to only a small subset of their consensus motifs on the genome. Here is an example from our Sox9 ChIP-seq study [61]: Among more than one million Sox consensus sites on the mouse genome, only less than $1 \%$ of them showed significantly enriched Sox9 binding in chondrocytes [61]. These observations strongly support the view that additional machinery restricts TF binding to the genome. Epigenetic regulations, which are mainly mediated by histone modification and DNA methylation, are thought 
to underlie the machinery $[62,63]$. The epigenetic landscape governs how and when key functional elements on the genome are available in order to achieve cell-type-specific expressions of a critical set of genes, determining cell fate and cell identity. The epigenetic landscape in the osteoblast differentiation process has been extensively studied. Most of the studies utilized an osteoblast induction culture of MSCs as a model system.

Although the epigenetic landscape is generally thought to be cell-type distinct, Meyer et al. demonstrated its flexibility in osteoblast differentiation from mouse bone marrow MSCs [30]. The epigenetic pattern of undifferentiated MSCs resembled that of osteogenic cells [30], suggesting their default fate of the osteoblast lineage; however, these patterns were rapidly altered near adipogenesisrelated genes during adipogenic differentiation [30]. Importantly, these cells exhibited an epigenetic plasticity between osteogenic and adipogenic conditions; even after commitment to one lineage, epigenetic profiles could be changed into those of the counterpart [30]. The plasticity of the epigenetic landscape may provide MSCs with flexibility in responding to multiple stimuli rather than rigidity of commitment to specific lineages, contributing to their wide range of functions in postnatal tissue repair and metabolism.

Consistent with the above results, Rauch et al. showed that the chromatin landscape of human MSCs (hMSCs) was similar to that of differentiating osteoblasts, and undifferentiated MSCs had more preestablished enhancers near osteoblast-related genes than near adipocyte-related genes [43]. In addition, preestablished enhancers were primarily involved in osteoblast differentiation from hMSCs, whereas de novo establishment and activation of enhancers was involved in adipocyte differentiation [43]. The latter process showed more dramatic transcriptional changes than the former one [43]. Thus, the epigenetic landscape in MSCs and their differentiation into the two lineages is likely to be conserved among species.

Which histone modifications are primarily responsible for the osteoblast-distinct epigenetic landscape? Wu et al. provided some clues that could help to answer this question through their 
analyses of osteoblast differentiation from mouse bone marrow MSCs [64]. First, H3K27me3, a histone mark associated with transcriptional repression, was generally lost upon osteoblast differentiation, but the mark was rarely gained on genes undergoing repression during the process. Wu et al. speculated that in osteoblast differentiation, transcriptional repression was induced by passive mechanisms such as the absence of activation marks such as H3K36me3 and H3K27ac, rather than an H3K27me3-mediated active mechanism [64]. Second, dynamic changes of gene expression were indeed correlated with $\mathrm{H} 3 \mathrm{~K} 36 \mathrm{me} 3$, which is associated with transcriptional elongation, and H3K27ac [64]. These data may suggest that H3K36me3 and H3K27ac contribute more to the construction of an osteoblast-distinct epigenetic landscape than H3K27me3. Such a conclusion is partially supported by a report that SET-domain-containing 2 (SETD2), a methyltransferase for $\mathrm{H} 3 \mathrm{~K} 36$, plays a key role in commitment of MSCs into the osteoblast lineage [65].

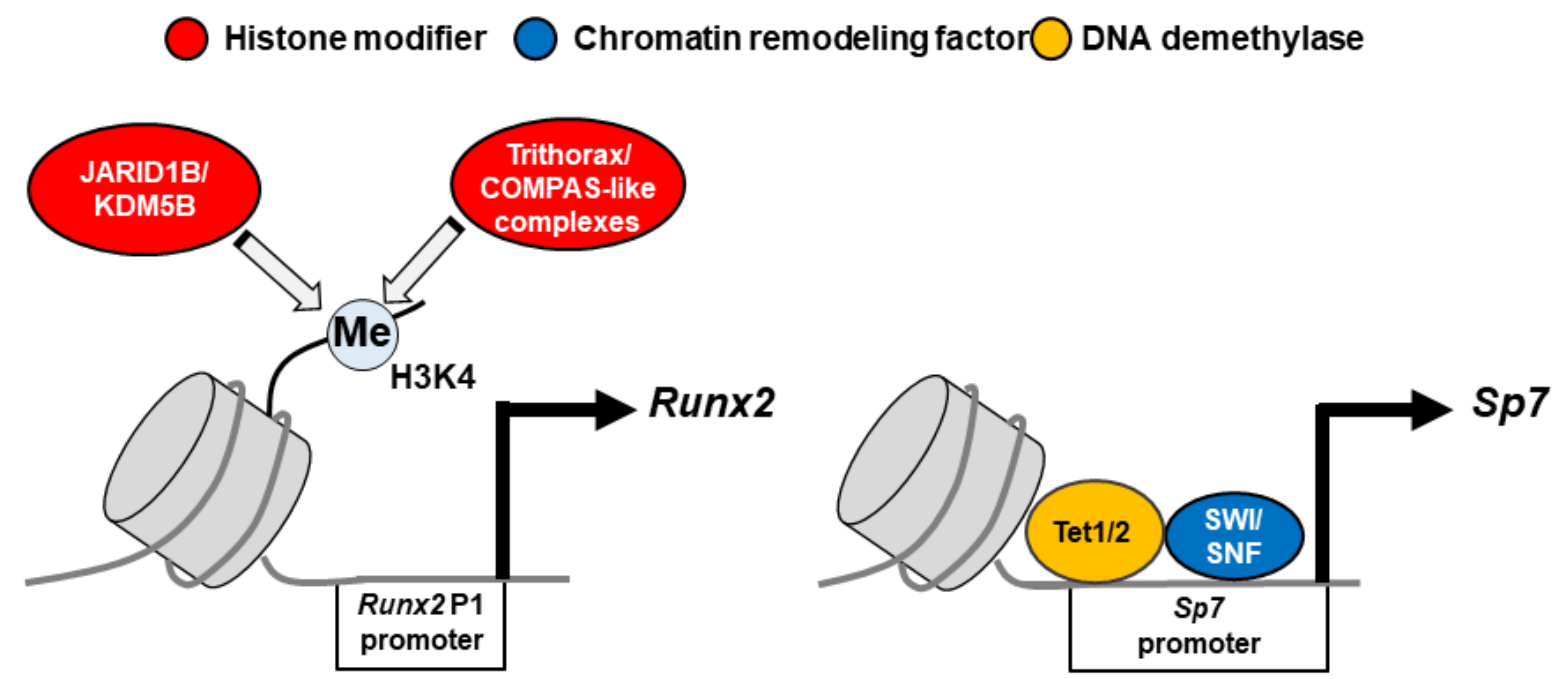

Fig. 2. Local actions of epigenetic regulators in the Runx 2 promoter and the Sp7 promoter. Local actions of histone modifiers, chromatin remodeling factors, and DNA demethylases play pivotal roles in transcriptions of Runx 2 and Sp7. Key actions identified mainly by in vitro biochemical analyses are illustrated. 
Agrawal Singh et al. proposed a role of $\mathrm{H} 3 \mathrm{~K} 27 \mathrm{me} 3$ in an early stage of osteoblast differentiation from hMSCs [66]. H3K27me3 was most strikingly lost in the ZBTB locus encoding the PLZF transcription factor, accompanied by gain of H3K27ac at the whole locus and H3K4me3 at its TSS, in immature osteoblasts [66]. PLZF was then highly expressed in response to osteogenic commitment and recruited to osteogenic enhancers, positively affecting lineage-specific gene expression [66]. Identification of EZH2 (enhancer of zeste homolog 2) as an inhibitor of osteoblast differentiation also highlights the involvement of $\mathrm{H} 3 \mathrm{~K} 27 \mathrm{me} 3$ in the osteoblast epigenetic landscape [67]. EZH2 is a polycomb group protein that catalyzes trimethylation of H3K27. Loss of function of EZH2 promoted osteoblast differentiation of hMSCs in vitro and led to multiple skeletal defects in mice [67].

In addition to EZH2 and SETD2, several chromatin modifiers have been implicated in osteoblast differentiation. Histone demethylase JARID1B/KDM5B was reported to regulate Runx2 expression, especially at the P1 promoter [68] (Fig. 2). The same group also demonstrated that Trithorax/COMPAS-like complexes containing Wdr5 and Utx modulated the transition from H3K4me1 to H3K4me3 at the P1 promoter to promote Runx2 expression [69] (Fig. 2). In terms of Runx2 activity, Mediator subunit MED23 was reported to act as a Runx2 cofactor by physical interaction [39] (Fig. 1). Epigenetic regulations in the expression of Sp7/Osterix were studied as well; Tet1/Tet2-mediated DNA demethylation, accompanied by SWI/SNF-dependent nucleosome remodeling, at the Sp7 promoter is critical for Sp7 expression [70] (Fig. 2). Lastly, the bromodomain-containing protein 4 (BRD4) is likely to play a role in osteoblast lineage commitment by occupying osteoblast-specific enhancers with AP-1, TEAD1, and C/EBP $\beta$ [71] (Fig. 1).

Thus, which histone modifications and chromatin modifiers are critical for the osteoblast program are still debated. Epigenetic status may be regulated by different sets of histone modifications/chromatin modifiers in different loci; the complex regulation is likely to constitute an osteoblast-distinct epigenetic landscape. 


\section{Concluding remarks}

Emerging genome-wide studies have provided insight into the gene regulatory landscape, which is made up of key transcriptional regulators and epigenetic regulations, in osteoblasts. However, current knowledge is limited to the regulatory landscape programmed by a few key transcriptional regulators. A clear overall picture of the landscape has not come into focus. Integrative analyses with osteogenic pathway-related TFs, their cofactors, and the epigenetic landscape will be required to understand the proper tuning of spatial and temporal gene regulatory networks in the dynamic process of osteoblast differentiation. In addition, extensive GWAS studies have identified links between the human genome and skeletal development or diseases [72, 73]. More than $90 \%$ of disease-associated loci have been identified outside of protein-coding regions, suggesting that the regulatory elements in non-coding regions, which are possibly involved in transcriptional activation, could be targets in next-generation genomics. Recent GWAS studies in the bone field have considered the higher order structure of the genome, including promoter interactome and threedimensional genomic landscape, in order to identify effector genes of GWAS loci [74, 75]. Accumulating knowledge on the gene regulatory landscape will provide a rich resource to connect the regulatory variants with human diseases. Further studies will not only enable us to elucidate the core gene regulatory network representing osteoblast identity and its dynamic differentiation process, but will also allow us to design genome-oriented therapeutic approaches for skeletal disorders.

\section{Author contributions}

Hironori Hojo and Shinsuke Ohba: Conception, literature review and writing of the manuscript.

\section{Declaration of competing interest}


The authors declare that they have no competing interests.

\section{Funding}

This work was supported by Grants-in-Aid for Scientific Research from the Japan Society for the Promotion of Science (JSPS: 17H04403, 17H05106, and 18K19636), Rising Star Awards from American Society for Bone and Mineral Research (to H.H. and S.O.), Mochida Memorial Foundation Research Grants (to H.H. and S.O.), and The Naito Grant for the Advancement of Natural Science (to S.O.).

\section{References}

[1] B.R. Olsen, A.M. Reginato, W. Wang, Bone development, Annu. Rev. Cell Dev. Biol., 16 (2000) 191-220.

[2] U.I. Chung, B. Lanske, K. Lee, E. Li, H. Kronenberg, The parathyroid hormone/parathyroid hormone-related peptide receptor coordinates endochondral bone development by directly controlling chondrocyte differentiation, Proc Natl Acad Sci U S A, 95 (1998) 13030-13035.

[3] U.I. Chung, E. Schipani, A.P. McMahon, H.M. Kronenberg, Indian hedgehog couples chondrogenesis to osteogenesis in endochondral bone development, J Clin Invest, 107 (2001) 295304.

[4] C. Maes, T. Kobayashi, M.K. Selig, S. Torrekens, S.I. Roth, S. Mackem, G. Carmeliet, H.M. Kronenberg, Osteoblast precursors, but not mature osteoblasts, move into developing and fractured bones along with invading blood vessels, Dev Cell, 19 (2010) 329-344.

[5] M.R. McClung, Sclerostin antibodies in osteoporosis: latest evidence and therapeutic potential, Ther Adv Musculoskelet Dis, 9 (2017) 263-270.

[6] P. Ducy, R. Zhang, V. Geoffroy, A.L. Ridall, G. Karsenty, Osf2/Cbfa1: a transcriptional activator of osteoblast differentiation, Cell, 89 (1997) 747-754. 
[7] K. Nakashima, X. Zhou, G. Kunkel, Z. Zhang, J.M. Deng, R.R. Behringer, B. de Crombrugghe, The novel zinc finger-containing transcription factor osterix is required for osteoblast differentiation and bone formation, Cell, 108 (2002) 17-29.

[8] T. Komori, H. Yagi, S. Nomura, A. Yamaguchi, K. Sasaki, K. Deguchi, Y. Shimizu, R.T. Bronson, Y.H. Gao, M. Inada, M. Sato, R. Okamoto, Y. Kitamura, S. Yoshiki, T. Kishimoto, Targeted disruption of $\mathrm{Cbfa} 1$ results in a complete lack of bone formation owing to maturational arrest of osteoblasts, Cell, 89 (1997) 755-764.

[9] B. Lee, K. Thirunavukkarasu, L. Zhou, L. Pastore, A. Baldini, J. Hecht, V. Geoffroy, P. Ducy, G. Karsenty, Missense mutations abolishing DNA binding of the osteoblast-specific transcription factor OSF2/CBFA1 in cleidocranial dysplasia, Nat Genet, 16 (1997) 307-310.

[10] F. Otto, A.P. Thornell, T. Crompton, A. Denzel, K.C. Gilmour, I.R. Rosewell, G.W. Stamp, R.S. Beddington, S. Mundlos, B.R. Olsen, P.B. Selby, M.J. Owen, Cbfa1, a candidate gene for cleidocranial dysplasia syndrome, is essential for osteoblast differentiation and bone development, Cell, 89 (1997) 765-771.

[11] S. Mundlos, F. Otto, C. Mundlos, J.B. Mulliken, A.S. Aylsworth, S. Albright, D. Lindhout, W.G. Cole, W. Henn, J.H. Knoll, M.J. Owen, R. Mertelsmann, B.U. Zabel, B.R. Olsen, Mutations involving the transcription factor CBFA1 cause cleidocranial dysplasia, Cell, 89 (1997) 773-779. [12] M. Fiscaletti, A. Biggin, B. Bennetts, K. Wong, J. Briody, V. Pacey, C. Birman, C.F. Munns, Novel variant in Sp7/Osx associated with recessive osteogenesis imperfecta with bone fragility and hearing impairment, Bone, 110 (2018) 66-75.

[13] P. Lapunzina, M. Aglan, S. Temtamy, J.A. Caparros-Martin, M. Valencia, R. Leton, V. MartinezGlez, R. Elhossini, K. Amr, N. Vilaboa, V.L. Ruiz-Perez, Identification of a frameshift mutation in Osterix in a patient with recessive osteogenesis imperfecta, Am J Hum Genet, 87 (2010) 110-114. [14] S.J. Rodda, A.P. McMahon, Distinct roles for Hedgehog and canonical Wnt signaling in specification, differentiation and maintenance of osteoblast progenitors, Development, 133 (2006) 
$3231-3244$.

[15] F.X. Long, Building strong bones: molecular regulation of the osteoblast lineage, Nat Rev Mol Cell Bio, 13 (2012) 27-38.

[16] C.H. Waddington, The strategy of the genes, Allen \& Unwin Ltd., London, 1957.

[17] R.J. Britten, E.H. Davidson, Gene regulation for higher cells: a theory, Science, 165 (1969) 349357.

[18] J. Banerji, S. Rusconi, W. Schaffner, Expression of a beta-globin gene is enhanced by remote SV40 DNA sequences, Cell, 27 (1981) 299-308.

[19] L.A. Lettice, S.J. Heaney, L.A. Purdie, L. Li, P. de Beer, B.A. Oostra, D. Goode, G. Elgar, R.E. Hill, E. de Graaff, A long-range Shh enhancer regulates expression in the developing limb and fin and is associated with preaxial polydactyly, Hum. Mol. Genet., 12 (2003) 1725-1735.

[20] P. Ducy, G. Karsenty, Two distinct osteoblast-specific cis-acting elements control expression of a mouse osteocalcin gene, Mol Cell Biol, 15 (1995) 1858-1869.

[21] G.S. Stein, J.B. Lian, A.J. van Wijnen, J.L. Stein, M. Montecino, A. Javed, S.K. Zaidi, D.W. Young, J.Y. Choi, S.M. Pockwinse, Runx2 control of organization, assembly and activity of the regulatory machinery for skeletal gene expression, Oncogene, 23 (2004) 4315-4329.

[22] A.M. Hakelien, J.C. Bryne, K.G. Harstad, S. Lorenz, J. Paulsen, J. Sun, T.S. Mikkelsen, O. Myklebost, L.A. Meza-Zepeda, The regulatory landscape of osteogenic differentiation, Stem Cells, 32 (2014) 2780-2793.

[23] M.B. Meyer, N.A. Benkusky, J.W. Pike, The RUNX2 cistrome in osteoblasts: characterization, down-regulation following differentiation, and relationship to gene expression, J Biol Chem, 289 (2014) 16016-16031.

[24] H. Wu, T.W. Whitfield, J.A. Gordon, J.R. Dobson, P.W. Tai, A.J. van Wijnen, J.L. Stein, G.S. Stein, J.B. Lian, Genomic occupancy of Runx2 with global expression profiling identifies a novel dimension to control of osteoblastogenesis, Genome biology, 15 (2014) R52. 
[25] M. Sato, E. Morii, T. Komori, H. Kawahata, M. Sugimoto, K. Terai, H. Shimizu, T. Yasui, H. Ogihara, N. Yasui, T. Ochi, Y. Kitamura, Y. Ito, S. Nomura, Transcriptional regulation of osteopontin gene in vivo by PEBP2alphaA/CBFA1 and ETS1 in the skeletal tissues, Oncogene, 17 (1998) 15171525.

[26] X. Wang, P.A. Manner, A. Horner, L. Shum, R.S. Tuan, G.H. Nuckolls, Regulation of MMP-13 expression by RUNX2 and FGF2 in osteoarthritic cartilage, Osteoarthritis Cartilage, 12 (2004) 963973.

[27] M.B. Meyer, N.A. Benkusky, J.W. Pike, Selective Distal Enhancer Control of the Mmp13 Gene Identified through Clustered Regularly Interspaced Short Palindromic Repeat (CRISPR) Genomic Deletions, J Biol Chem, 290 (2015) 11093-11107.

[28] M. Hirata, F. Kugimiya, A. Fukai, S. Ohba, N. Kawamura, T. Ogasawara, Y. Kawasaki, T. Saito, F. Yano, T. Ikeda, K. Nakamura, U.I. Chung, H. Kawaguchi, C/EBPbeta Promotes transition from proliferation to hypertrophic differentiation of chondrocytes through transactivation of p57, PLoS One, 4 (2009) e4543.

[29] H. Tominaga, S. Maeda, M. Hayashi, S. Takeda, S. Akira, S. Komiya, T. Nakamura, H. Akiyama, T. Imamura, CCAAT/enhancer-binding protein beta promotes osteoblast differentiation by enhancing Runx2 activity with ATF4, Mol Biol Cell, 19 (2008) 5373-5386.

[30] M.B. Meyer, N.A. Benkusky, B. Sen, J. Rubin, J.W. Pike, Epigenetic Plasticity Drives Adipogenic and Osteogenic Differentiation of Marrow-derived Mesenchymal Stem Cells, J Biol Chem, 291 (2016) 17829-17847.

[31] E.Z. Kvon, G. Stampfel, J.O. Yanez-Cuna, B.J. Dickson, A. Stark, HOT regions function as patterned developmental enhancers and have a distinct cis-regulatory signature, Genes Dev, 26 (2012) 908-913.

[32] M. Kundu, A. Javed, J.P. Jeon, A. Horner, L. Shum, M. Eckhaus, M. Muenke, J.B. Lian, Y. Yang, G.H. Nuckolls, G.S. Stein, P.P. Liu, Cbfbeta interacts with Runx2 and has a critical role in 
bone development, Nat Genet, 32 (2002) 639-644.

[33] C.A. Yoshida, T. Furuichi, T. Fujita, R. Fukuyama, N. Kanatani, S. Kobayashi, M. Satake, K. Takada, T. Komori, Core-binding factor beta interacts with Runx2 and is required for skeletal development, Nat Genet, 32 (2002) 633-638.

[34] P. Bialek, B. Kern, X. Yang, M. Schrock, D. Sosic, N. Hong, H. Wu, K. Yu, D.M. Ornitz, E.N. Olson, M.J. Justice, G. Karsenty, A twist code determines the onset of osteoblast differentiation, Dev Cell, 6 (2004) 423-435.

[35] S. Kim, T. Koga, M. Isobe, B.E. Kern, T. Yokochi, Y.E. Chin, G. Karsenty, T. Taniguchi, H. Takayanagi, Stat1 functions as a cytoplasmic attenuator of Runx2 in the transcriptional program of osteoblast differentiation, Genes Dev, 17 (2003) 1979-1991.

[36] D.C. Jones, M.N. Wein, M. Oukka, J.G. Hofstaetter, M.J. Glimcher, L.H. Glimcher, Regulation of adult bone mass by the zinc finger adapter protein Schnurri-3, Science, 312 (2006) 1223-1227. [37] G. Dobreva, M. Chahrour, M. Dautzenberg, L. Chirivella, B. Kanzler, I. Farinas, G. Karsenty, R. Grosschedl, SATB2 is a multifunctional determinant of craniofacial patterning and osteoblast differentiation, Cell, 125 (2006) 971-986.

[38] J.H. Hong, E.S. Hwang, M.T. McManus, A. Amsterdam, Y. Tian, R. Kalmukova, E. Mueller, T. Benjamin, B.M. Spiegelman, P.A. Sharp, N. Hopkins, M.B. Yaffe, TAZ, a transcriptional modulator of mesenchymal stem cell differentiation, Science, 309 (2005) 1074-1078.

[39] Z. Liu, X. Yao, G. Yan, Y. Xu, J. Yan, W. Zou, G. Wang, Mediator MED23 cooperates with RUNX2 to drive osteoblast differentiation and bone development, Nat Commun, 7 (2016) 11149. [40] X. Zhang, K. Ting, C.M. Bessette, C.T. Culiat, S.J. Sung, H. Lee, F. Chen, J. Shen, J.J. Wang, S. Kuroda, C. Soo, Nell-1, a key functional mediator of Runx2, partially rescues calvarial defects in Runx2(+/-) mice, J Bone Miner Res, 26 (2011) 777-791.

[41] E. Hesse, H. Saito, R. Kiviranta, D. Correa, K. Yamana, L. Neff, D. Toben, G. Duda, A. Atfi, V. Geoffroy, W.C. Horne, R. Baron, Zfp521 controls bone mass by HDAC3-dependent attenuation of 
Runx2 activity, J Cell Biol, 191 (2010) 1271-1283.

[42] I. Tosa, D. Yamada, M. Yasumatsu, E. Hinoi, M. Ono, T. Oohashi, T. Kuboki, T. Takarada, Postnatal Runx2 deletion leads to low bone mass and adipocyte accumulation in mice bone tissues, Biochem Biophys Res Commun, 516 (2019) 1229-1233.

[43] A. Rauch, A.K. Haakonsson, J.G.S. Madsen, M. Larsen, I. Forss, M.R. Madsen, E.L. Van Hauwaert, C. Wiwie, N.Z. Jespersen, M. Tencerova, R. Nielsen, B.D. Larsen, R. Rottger, J. Baumbach, C. Scheele, M. Kassem, S. Mandrup, Osteogenesis depends on commissioning of a network of stem cell transcription factors that act as repressors of adipogenesis, Nat Genet, 51 (2019) 716-727.

[44] H. Hojo, S. Ohba, X. He, L.P. Lai, A.P. McMahon, Sp7/Osterix Is Restricted to Bone-Forming Vertebrates where It Acts as a Dlx Co-factor in Osteoblast Specification, Dev Cell, 37 (2016) 238253.

[45] S. Safe, M. Abdelrahim, Sp transcription factor family and its role in cancer, Eur. J. Cancer, 41 (2005) 2438-2448.

[46] J. Wang, J. Zhuang, S. Iyer, X. Lin, T.W. Whitfield, M.C. Greven, B.G. Pierce, X. Dong, A. Kundaje, Y. Cheng, O.J. Rando, E. Birney, R.M. Myers, W.S. Noble, M. Snyder, Z. Weng, Sequence features and chromatin structure around the genomic regions bound by 119 human transcription factors, Genome Res., 22 (2012) 1798-1812.

[47] G. Terrados, F. Finkernagel, B. Stielow, D. Sadic, J. Neubert, O. Herdt, M. Krause, M. Scharfe, M. Jarek, G. Suske, Genome-wide localization and expression profiling establish $\mathrm{Sp} 2$ as a sequencespecific transcription factor regulating vitally important genes, Nucleic Acids Res, 40 (2012) 78447857.

[48] M.W. Kennedy, R.B. Chalamalasetty, S. Thomas, R.J. Garriock, P. Jailwala, T.P. Yamaguchi, Sp5 and Sp8 recruit beta-catenin and Tcf1-Lef1 to select enhancers to activate Wnt target gene transcription, Proc Natl Acad Sci U S A, 113 (2016) 3545-3550. 
[49] M.A. Hume, L.A. Barrera, S.S. Gisselbrecht, M.L. Bulyk, UniPROBE, update 2015: new tools and content for the online database of protein-binding microarray data on protein-DNA interactions, Nucleic Acids Res, 43 (2015) D117-122.

[50] E. Wingender, T. Schoeps, J. Donitz, TFClass: an expandable hierarchical classification of human transcription factors, Nucleic Acids Res, 41 (2013) D165-170.

[51] M.Q. Hassan, A. Javed, M.I. Morasso, J. Karlin, M. Montecino, A.J. van Wijnen, G.S. Stein, J.L. Stein, J.B. Lian, Dlx3 transcriptional regulation of osteoblast differentiation: temporal recruitment of Msx2, Dlx3, and Dlx5 homeodomain proteins to chromatin of the osteocalcin gene, Mol Cell Biol, 24 (2004) 9248-9261.

[52] H. Li, I. Marijanovic, M.S. Kronenberg, I. Erceg, M.L. Stover, D. Velonis, M. Mina, J.G. Heinrich, S.E. Harris, W.B. Upholt, I. Kalajzic, A.C. Lichtler, Expression and function of Dlx genes in the osteoblast lineage, Dev Biol, 316 (2008) 458-470.

[53] T. Tadic, M. Dodig, I. Erceg, I. Marijanovic, M. Mina, Z. Kalajzic, D. Velonis, M.S. Kronenberg, R.A. Kosher, D. Ferrari, A.C. Lichtler, Overexpression of Dlx 5 in chicken calvarial cells accelerates osteoblastic differentiation, J Bone Miner Res, 17 (2002) 1008-1014. [54] N. Artigas, C. Urena, E. Rodriguez-Carballo, J.L. Rosa, F. Ventura, Mitogen-activated protein kinase (MAPK)-regulated interactions between Osterix and Runx2 are critical for the transcriptional osteogenic program, J Biol Chem, 289 (2014) 27105-27117.

[55] T. Koga, Y. Matsui, M. Asagiri, T. Kodama, B. de Crombrugghe, K. Nakashima, H. Takayanagi, NFAT and Osterix cooperatively regulate bone formation, Nat Med, 11 (2005) 880-885.

[56] T. Kawane, H. Komori, W. Liu, T. Moriishi, T. Miyazaki, M. Mori, Y. Matsuo, Y. Takada, S. Izumi, Q. Jiang, R. Nishimura, Y. Kawai, T. Komori, Dlx5 and mef2 regulate a novel runx2 enhancer for osteoblast-specific expression, J Bone Miner Res, 29 (2014) 1960-1969.

[57] A. Bedalov, R. Salvatori, M. Dodig, M.S. Kronenberg, B. Kapural, Z. Bogdanovic, B.E. Kream, C.O. Woody, S.H. Clark, K. Mack, et al., Regulation of COL1A1 expression in type I collagen 
producing tissues: identification of a 49 base pair region which is required for transgene expression in bone of transgenic mice, J Bone Miner Res, 10 (1995) 1443-1451.

[58] R. Nishimura, M. Wakabayashi, K. Hata, T. Matsubara, S. Honma, S. Wakisaka, H. Kiyonari, G. Shioi, A. Yamaguchi, N. Tsumaki, H. Akiyama, T. Yoneda, Osterix regulates calcification and degradation of chondrogenic matrices through matrix metalloproteinase 13 (MMP13) expression in association with transcription factor Runx2 during endochondral ossification, J Biol Chem, 287 (2012) 33179-33190.

[59] H. Rashid, C. Ma, H. Chen, H. Wang, M.Q. Hassan, K. Sinha, B. de Crombrugghe, A. Javed, Sp7 and Runx2 molecular complex synergistically regulate expression of target genes, Connect. Tissue Res., 55 Suppl 1 (2014) 83-87.

[60] Y. Han, C.Y. Kim, H. Cheong, K.Y. Lee, Osterix represses adipogenesis by negatively regulating PPARgamma transcriptional activity, Sci Rep, 6 (2016) 35655.

[61] S. Ohba, X. He, H. Hojo, A.P. McMahon, Distinct Transcriptional Programs Underlie Sox9 Regulation of the Mammalian Chondrocyte, Cell reports, 12 (2015) 229-243.

[62] E. Calo, J. Wysocka, Modification of enhancer chromatin: what, how, and why?, Mol Cell, 49 (2013) 825-837.

[63] J.L. Plank, A. Dean, Enhancer function: mechanistic and genome-wide insights come together, Mol Cell, 55 (2014) 5-14.

[64] H. Wu, J.A. Gordon, T.W. Whitfield, P.W. Tai, A.J. van Wijnen, J.L. Stein, G.S. Stein, J.B. Lian, Chromatin dynamics regulate mesenchymal stem cell lineage specification and differentiation to osteogenesis, Biochim Biophys Acta Gene Regul Mech, 1860 (2017) 438-449.

[65] L. Wang, N. Niu, L. Li, R. Shao, H. Ouyang, W. Zou, H3K36 trimethylation mediated by SETD2 regulates the fate of bone marrow mesenchymal stem cells, PLoS Biol, 16 (2018) e2006522. [66] S. Agrawal Singh, M. Lerdrup, A.R. Gomes, H.J. van de Werken, J. Vilstrup Johansen, R. Andersson, A. Sandelin, K. Helin, K. Hansen, PLZF targets developmental enhancers for activation 
during osteogenic differentiation of human mesenchymal stem cells, Elife, 8 (2019).

[67] A. Dudakovic, E.T. Camilleri, F. Xu, S.M. Riester, M.E. McGee-Lawrence, E.W. Bradley, C.R. Paradise, E.A. Lewallen, R. Thaler, D.R. Deyle, A.N. Larson, D.G. Lewallen, A.B. Dietz, G.S. Stein, M.A. Montecino, J.J. Westendorf, A.J. van Wijnen, Epigenetic Control of Skeletal Development by the Histone Methyltransferase Ezh2, J Biol Chem, 290 (2015) 27604-27617.

[68] A. Rojas, R. Aguilar, B. Henriquez, J.B. Lian, J.L. Stein, G.S. Stein, A.J. van Wijnen, B. van Zundert, M.L. Allende, M. Montecino, Epigenetic Control of the Bone-master Runx2 Gene during Osteoblast-lineage Commitment by the Histone Demethylase JARID1B/KDM5B, J Biol Chem, 290 (2015) 28329-28342.

[69] A. Rojas, H. Sepulveda, B. Henriquez, R. Aguilar, T. Opazo, G. Nardocci, F. Bustos, J.B. Lian, J.L. Stein, G.S. Stein, B. van Zundert, A.J. van Wijnen, M.L. Allende, M. Montecino, MllCOMPASS complexes mediate H3K4me3 enrichment and transcription of the osteoblast master gene Runx2/p57 in osteoblasts, J. Cell. Physiol., 234 (2019) 6244-6253.

[70] H. Sepulveda, A. Villagra, M. Montecino, Tet-Mediated DNA Demethylation Is Required for SWI/SNF-Dependent Chromatin Remodeling and Histone-Modifying Activities That Trigger Expression of the Sp7 Osteoblast Master Gene during Mesenchymal Lineage Commitment, Mol Cell Biol, 37 (2017).

[71] Z. Najafova, R. Tirado-Magallanes, M. Subramaniam, T. Hossan, G. Schmidt, S. Nagarajan, S.J. Baumgart, V.K. Mishra, U. Bedi, E. Hesse, S. Knapp, J.R. Hawse, S.A. Johnsen, BRD4 localization to lineage-specific enhancers is associated with a distinct transcription factor repertoire, Nucleic Acids Res, 45 (2017) 127-141.

[72] A.R. Wood, T. Esko, J. Yang, S. Vedantam, T.H. Pers, S. Gustafsson, A.Y. Chu, K. Estrada, J. Luan, Z. Kutalik, N. Amin, M.L. Buchkovich, D.C. Croteau-Chonka, F.R. Day, Y. Duan, T. Fall, R. Fehrmann, T. Ferreira, A.U. Jackson, J. Karjalainen, K.S. Lo, A.E. Locke, R. Magi, E. Mihailov, E. Porcu, J.C. Randall, A. Scherag, A.A. Vinkhuyzen, H.J. Westra, T.W. Winkler, T. Workalemahu, J.H. 
Zhao, D. Absher, E. Albrecht, D. Anderson, J. Baron, M. Beekman, A. Demirkan, G.B. Ehret, B. Feenstra, M.F. Feitosa, K. Fischer, R.M. Fraser, A. Goel, J. Gong, A.E. Justice, S. Kanoni, M.E. Kleber, K. Kristiansson, U. Lim, V. Lotay, J.C. Lui, M. Mangino, I. Mateo Leach, C. Medina-Gomez, M.A. Nalls, D.R. Nyholt, C.D. Palmer, D. Pasko, S. Pechlivanis, I. Prokopenko, J.S. Ried, S. Ripke, D. Shungin, A. Stancakova, R.J. Strawbridge, Y.J. Sung, T. Tanaka, A. Teumer, S. Trompet, S.W. van der Laan, J. van Setten, J.V. Van Vliet-Ostaptchouk, Z. Wang, L. Yengo, W. Zhang, U. Afzal, J. Arnlov, G.M. Arscott, S. Bandinelli, A. Barrett, C. Bellis, A.J. Bennett, C. Berne, M. Bluher, J.L. Bolton, Y. Bottcher, H.A. Boyd, M. Bruinenberg, B.M. Buckley, S. Buyske, I.H. Caspersen, P.S. Chines, R. Clarke, S. Claudi-Boehm, M. Cooper, E.W. Daw, P.A. De Jong, J. Deelen, G. Delgado, J.C. Denny, R. Dhonukshe-Rutten, M. Dimitriou, A.S. Doney, M. Dorr, N. Eklund, E. Eury, L. Folkersen, M.E. Garcia, F. Geller, V. Giedraitis, A.S. Go, H. Grallert, T.B. Grammer, J. Grassler, H. Gronberg, L.C. de Groot, C.J. Groves, J. Haessler, P. Hall, T. Haller, G. Hallmans, A. Hannemann, C.A. Hartman, M. Hassinen, C. Hayward, N.L. Heard-Costa, Q. Helmer, G. Hemani, A.K. Henders, H.L. Hillege, M.A. Hlatky, W. Hoffmann, P. Hoffmann, O. Holmen, J.J. Houwing-Duistermaat, T. Illig, A. Isaacs, A.L. James, J. Jeff, B. Johansen, A. Johansson, J. Jolley, T. Juliusdottir, J. Junttila, A.N. Kho, L. Kinnunen, N. Klopp, T. Kocher, W. Kratzer, P. Lichtner, L. Lind, J. Lindstrom, S. Lobbens, M. Lorentzon, Y. Lu, V. Lyssenko, P.K. Magnusson, A. Mahajan, M. Maillard, W.L. McArdle, C.A. McKenzie, S. McLachlan, P.J. McLaren, C. Menni, S. Merger, L. Milani, A. Moayyeri, K.L. Monda, M.A. Morken, G. Muller, M. Muller-Nurasyid, A.W. Musk, N. Narisu, M. Nauck, I.M. Nolte, M.M. Nothen, L. Oozageer, S. Pilz, N.W. Rayner, F. Renstrom, N.R. Robertson, L.M. Rose, R. Roussel, S. Sanna, H. Scharnagl, S. Scholtens, F.R. Schumacher, H. Schunkert, R.A. Scott, J. Sehmi, T. Seufferlein, J. Shi, K. Silventoinen, J.H. Smit, A.V. Smith, J. Smolonska, A.V. Stanton, K. Stirrups, D.J. Stott, H.M. Stringham, J. Sundstrom, M.A. Swertz, A.C. Syvanen, B.O. Tayo, G. Thorleifsson, J.P. Tyrer, S. van Dijk, N.M. van Schoor, N. van der Velde, D. van Heemst, F.V. van Oort, S.H. Vermeulen, N. Verweij, J.M. Vonk, L.L. Waite, M. Waldenberger, R. Wennauer, 
L.R. Wilkens, C. Willenborg, T. Wilsgaard, M.K. Wojczynski, A. Wong, A.F. Wright, Q. Zhang, D. Arveiler, S.J. Bakker, J. Beilby, R.N. Bergman, S. Bergmann, R. Biffar, J. Blangero, D.I. Boomsma, S.R. Bornstein, P. Bovet, P. Brambilla, M.J. Brown, H. Campbell, M.J. Caulfield, A. Chakravarti, R. Collins, F.S. Collins, D.C. Crawford, L.A. Cupples, J. Danesh, U. de Faire, H.M. den Ruijter, R. Erbel, J. Erdmann, J.G. Eriksson, M. Farrall, E. Ferrannini, J. Ferrieres, I. Ford, N.G. Forouhi, T. Forrester, R.T. Gansevoort, P.V. Gejman, C. Gieger, A. Golay, O. Gottesman, V. Gudnason, U. Gyllensten, D.W. Haas, A.S. Hall, T.B. Harris, A.T. Hattersley, A.C. Heath, C. Hengstenberg, A.A. Hicks, L.A. Hindorff, A.D. Hingorani, A. Hofman, G.K. Hovingh, S.E. Humphries, S.C. Hunt, E. Hypponen, K.B. Jacobs, M.R. Jarvelin, P. Jousilahti, A.M. Jula, J. Kaprio, J.J. Kastelein, M. Kayser, F. Kee, S.M. Keinanen-Kiukaanniemi, L.A. Kiemeney, J.S. Kooner, C. Kooperberg, S. Koskinen, P. Kovacs, A.T. Kraja, M. Kumari, J. Kuusisto, T.A. Lakka, C. Langenberg, L. Le Marchand, T. Lehtimaki, S. Lupoli, P.A. Madden, S. Mannisto, P. Manunta, A. Marette, T.C. Matise, B. McKnight, T. Meitinger, F.L. Moll, G.W. Montgomery, A.D. Morris, A.P. Morris, J.C. Murray, M. Nelis, C. Ohlsson, A.J. Oldehinkel, K.K. Ong, W.H. Ouwehand, G. Pasterkamp, A. Peters, P.P. Pramstaller, J.F. Price, L. Qi, O.T. Raitakari, T. Rankinen, D.C. Rao, T.K. Rice, M. Ritchie, I. Rudan, V. Salomaa, N.J. Samani, J. Saramies, M.A. Sarzynski, P.E. Schwarz, S. Sebert, P. Sever, A.R. Shuldiner, J. Sinisalo, V. Steinthorsdottir, R.P. Stolk, J.C. Tardif, A. Tonjes, A. Tremblay, E. Tremoli, J. Virtamo, M.C. Vohl, R. Electronic Medical, C. Genomics, M.I. Consortium, P. Consortium, S. LifeLines Cohort, P. Amouyel, F.W. Asselbergs, T.L. Assimes, M. Bochud, B.O. Boehm, E. Boerwinkle, E.P. Bottinger, C. Bouchard, S. Cauchi, J.C. Chambers, S.J. Chanock, R.S. Cooper, P.I. de Bakker, G. Dedoussis, L. Ferrucci, P.W. Franks, P. Froguel, L.C. Groop, C.A. Haiman, A. Hamsten, M.G. Hayes, J. Hui, D.J. Hunter, K. Hveem, J.W. Jukema, R.C. Kaplan, M. Kivimaki, D. Kuh, M. Laakso, Y. Liu, N.G. Martin, W. Marz, M. Melbye, S. Moebus, P.B. Munroe, I. Njolstad, B.A. Oostra, C.N. Palmer, N.L. Pedersen, M. Perola, L. Perusse, U. Peters, J.E. Powell, C. Power, T. Quertermous, R. Rauramaa, E. Reinmaa, P.M. Ridker, F. Rivadeneira, J.I. Rotter, T.E. Saaristo, D. Saleheen, D. Schlessinger, P.E. 
Slagboom, H. Snieder, T.D. Spector, K. Strauch, M. Stumvoll, J. Tuomilehto, M. Uusitupa, P. van der Harst, H. Volzke, M. Walker, N.J. Wareham, H. Watkins, H.E. Wichmann, J.F. Wilson, P. Zanen, P. Deloukas, I.M. Heid, C.M. Lindgren, K.L. Mohlke, E.K. Speliotes, U. Thorsteinsdottir, I. Barroso, C.S. Fox, K.E. North, D.P. Strachan, J.S. Beckmann, S.I. Berndt, M. Boehnke, I.B. Borecki, M.I. McCarthy, A. Metspalu, K. Stefansson, A.G. Uitterlinden, C.M. van Duijn, L. Franke, C.J. Willer, A.L. Price, G. Lettre, R.J. Loos, M.N. Weedon, E. Ingelsson, J.R. O'Connell, G.R. Abecasis, D.I. Chasman, M.E. Goddard, P.M. Visscher, J.N. Hirschhorn, T.M. Frayling, Defining the role of common variation in the genomic and biological architecture of adult human height, Nat Genet, 46 (2014) 1173-1186.

[73] J.P. Kemp, C. Medina-Gomez, K. Estrada, B. St Pourcain, D.H. Heppe, N.M. Warrington, L. Oei, S.M. Ring, C.J. Kruithof, N.J. Timpson, L.E. Wolber, S. Reppe, K. Gautvik, E. Grundberg, B. Ge, B. van der Eerden, J. van de Peppel, M.A. Hibbs, C.L. Ackert-Bicknell, K. Choi, D.L. Koller, M.J. Econs, F.M. Williams, T. Foroud, M.C. Zillikens, C. Ohlsson, A. Hofman, A.G. Uitterlinden, G. Davey Smith, V.W. Jaddoe, J.H. Tobias, F. Rivadeneira, D.M. Evans, Phenotypic dissection of bone mineral density reveals skeletal site specificity and facilitates the identification of novel loci in the genetic regulation of bone mass attainment, PLoS Genet, 10 (2014) e1004423.

[74] A. Chesi, Y. Wagley, M.E. Johnson, E. Manduchi, C. Su, S. Lu, M.E. Leonard, K.M. Hodge, J.A. Pippin, K.D. Hankenson, A.D. Wells, S.F.A. Grant, Genome-scale Capture C promoter interactions implicate effector genes at GWAS loci for bone mineral density, Nat Commun, 10 (2019) 1260.

[75] J.A. Morris, J.P. Kemp, S.E. Youlten, L. Laurent, J.G. Logan, R.C. Chai, N.A. Vulpescu, V. Forgetta, A. Kleinman, S.T. Mohanty, C.M. Sergio, J. Quinn, L. Nguyen-Yamamoto, A.L. Luco, J. Vijay, M.M. Simon, A. Pramatarova, C. Medina-Gomez, K. Trajanoska, E.J. Ghirardello, N.C. Butterfield, K.F. Curry, V.D. Leitch, P.C. Sparkes, A.T. Adoum, N.S. Mannan, D.S.K. Komla-Ebri, A.S. Pollard, H.F. Dewhurst, T.A.D. Hassall, M.G. Beltejar, T. andMe Research, D.J. Adams, S.M. 
Vaillancourt, S. Kaptoge, P. Baldock, C. Cooper, J. Reeve, E.E. Ntzani, E. Evangelou, C. Ohlsson, D. Karasik, F. Rivadeneira, D.P. Kiel, J.H. Tobias, C.L. Gregson, N.C. Harvey, E. Grundberg, D. Goltzman, D.J. Adams, C.J. Lelliott, D.A. Hinds, C.L. Ackert-Bicknell, Y.H. Hsu, M.T. Maurano, P.I. Croucher, G.R. Williams, J.H.D. Bassett, D.M. Evans, J.B. Richards, An atlas of genetic influences on osteoporosis in humans and mice, Nat Genet, 51 (2019) 258-266. 